Remark. Using the same method as in the proof of Lemma 11 we can prove that for each finite group $B$ we have $H_{3}(B)+H_{3}(B) \supset B \otimes B$. $B y$ this relation and the first part of the proof of Theorem 14 it follows that a class is weakly complete even in the case when the group $H_{3}(A)$ is in $e$ for any group $A$ from $e$. This last property is then equivalent to the perfectness of $e$.

For any integer $n \geqslant 1$ and any (abelian if $n>1$ ) group $A$ the groups $H_{m}(A, n)$ are defined as homology groups of the Eilenberg-MacLane complex $K(A, n)$. If $n=1$ then $H_{m}(A, 1)=H_{m}(A)$. Theorem 14 and Proposition 6.11 of [6] (p. 304) imply

THEOREM 15. If $e$ is a weakly complete class and a group $A$ is in $e$, then all the groups $H_{m}(A, n), m>0$, are in $e$.

\title{
References
}

[1] S. Balcerzyk, On classes of abelian groups, Bull. Acad. Polon. Sci., Ċ. III, 9 (1961), pp. $327-329$

[2] H. Cartan, S. Eilenberg, Homological Algebra, Princeton 1956.

[3] S. Eilenberg, S. MacLane, Relations between homology and homotopy groups of spaces, Annals of Math. 46 (1945), pp. 480-509.

[4]' L. Fuchs, Abelian groups, Budapest 1958.

[5] G. Hochschild, J.-P. Serre, Cohomology of groups extensions, Trans. Amer. Math. Soc. 74 (1953), pp. 110-134.

[6] S. T. Hu, Homotopy theory, New York 1959.

[7] A. G. Kurosh, The theory of groups, New York 1955.

[8] J.-P. Serre, Groupes d'homotopie et classes de groupes abeliens, Ann. of Math. 58 (1953), pp. 258-294.

INSTYTUT MATEMATYCZNY POLSKIEJ AKADEMII NAUK

INSTITUTE OF MATHEMATICS OF THE POLISH ACADEMY OF SCIENCES

Reşu par la Rédaction le 12. 9. 1961

\section{A functional conception of snake-like continua}

by

\section{J. Mioduszewski (Wroclaw)}

It is known [5] that snake-like continua (in short $\mathrm{SC}$ ) in the sense of Bing [2] may be regarded as inverse limit spaces of arcs (closed intervals) with projections which are continuous mappings onto.

This method of construction will be applied here to an important class of SC, viz. to the hereditarily indecomposable SC. The existence of hereditarily indecomposable SC was shown by Knaster [6]. Bing called them pseudoarcs and proved their homeomorphism to one another [3]. I prove that every SC is a continuous image of the pseudoarc; therefore, the pseudoarc will be called here the universal snake-like continuum (in short USC). This result seems to be a consequence of a certain theorem of Bing (see [2], Theorem 5 and Lehner [7], Theorem 1), but I intend to use this opportunity to exemplify how the method of inverse limits can be applied to this kind of problems. Therefore, my construction does not resort to Bing's geometrical method using crookedness. I use particularly the uniformization theorem of Sikorski and Zarankiewicz (see [9] and [11]) concerning continuous mappings- of the closed interval onto itself.

Waraszkiewicz [12] showed that there exists no continuum of which an arbitrary continuum would be a continuous image, i.e. would be universal for the class of all continua. Henceforth, the following question seems to be interesting: how large is the class of continua for which USC is still universal?

§ 1. Preliminaries. We consider $\mathrm{SC}$ as inverse limit spaces $X=\lim _{\longleftarrow}\left\{X_{n}, \pi_{n}^{m}\right\}$ of ares $X_{n}$ with projections $\pi_{n}^{m}: X_{m} \rightarrow X_{n}, m \geqslant n$, $m, n=1,2, \ldots$, which are continuous and onto $\left(\pi_{n}^{n}\right.$ are assumed to be identities). We assume, for convenience, that $X_{n}$ are closed unit intervals, i.e. $X_{n}=\left\{x_{n}: 0 \leqslant x_{n} \leqslant 1\right\}$. Consequently, $\mathrm{SC}$ are 1 -dimensional metric continua (see theorems on inverse limits in [4]). It is also known that SC are imbeddable into the plane (see [2] and [5]). (1).

(1) A quite elementary proof of the last proposition is as follows.

The inverse limit does not change if we subtitute (even in infinitely many places) $\pi_{n}^{n+1} h_{n}$ for $\pi_{n}^{n+1}$, where $h_{n}$ is a homeomorphism of $X_{n+1}$ onto itself. Furthermore, every 
Let $I$ be the closed unit interval. Let $f, g: I \rightarrow I$ be continuous and onto. We write $f \prec g$ if there exists an $\alpha: I \rightarrow I$, also continuous and onto, such that $f \alpha=g$. Let $S$ be a class of mappings $f: I \rightarrow I$ continuous and onto. We say that $g$ is a majorant for $S$ if $f \prec I$ for every $f \in S$. We say that $g$ is a strong majorant for $S$ if for every $f^{\prime}, f^{\prime \prime} \in S$ we have $f^{\prime \prime} \prec f^{\prime} g$. Relation $\prec$ was investigated in [9].

Let $I^{\prime}$ be a triangulation (division) of $I$. Simplices (segments) of $I^{\prime}$ are assumed to be equal. We denote by $\eta\left(I^{\prime}\right)$ the number of segments in $I^{\prime}$. Consequently, mesh $I^{\prime}=1 / \eta\left(I^{\prime}\right)$. The closed intervals which are the sums of segments of $I^{\prime}$ will be called subintervals of $I^{\prime}$.

Let $I^{\prime \prime}$ be another division of $I$ of this kind. Consider the class $\left[I^{\prime \prime} \rightarrow I^{\prime}\right]$ of simplicial (piece-wise linear) mappings from $I^{\prime \prime}$ onto $I^{\prime}$. This class is, of course, finite. It is non-empty if $\eta\left(I^{\prime \prime}\right) \geqslant \eta\left(I^{\prime}\right)$.

LEMMA 1. There exist a division $I^{\prime \prime \prime}$ of $I$ and a mapping $g \in\left[I^{\prime \prime \prime} \rightarrow I^{\prime \prime}\right]$ which is a strong majorant for $\left[I^{\prime \prime} \rightarrow I^{\prime}\right]$.

inverse system $\left\{X_{n}, \pi_{n}^{m}\right\}$, where $X_{n}$ are are and $\pi_{n}^{m}$ are continuous mappings onto, may be regarded as a subsystem of an inverse system $\left\{E_{n}, \pi_{n}^{* m}\right\}$, where $E_{n}$ are Euclidean planes and $\pi_{n}^{* m}$, which are continuous and onto, are defined as follows.

Let $g_{n}$ be a homeomorphism of $X_{n+1}$ onto the graph $Y_{n}$ of $\pi_{n}^{n+1}$ given by the formula $g_{n}\left(x_{n+1}\right)=\left(x_{n+1}, \pi_{n}^{n+1}\left(x_{n+1}\right)\right)=\left(x_{n+1}, x_{n}\right)$. Consider the inverse system

$$
X_{1} \leftarrow \ldots \leftarrow X_{n} \underset{\pi_{n}^{n+1} g_{n}^{-1}}{\leftarrow} \boldsymbol{Y}_{n} \leftarrow X_{n+1} \leftarrow \ldots,
$$

having the same limit as $\left\{X_{n}, \pi_{n}^{m}\right\}$. Now, we extend mappings $\pi_{n}^{n+1} g_{n}^{-1}$ to mappings $\pi_{n}^{* n+1}$ which map the plane onto the plane as follows:

where

$$
\pi_{n}^{* n+1}\left(x_{n+1}, x_{n}\right)=\left(\xi_{n+1}, x_{n}\right),
$$

$$
\xi_{n+1}=\left\{\begin{array}{ccl}
x_{n+1} & \text { for } & x_{n+1} \geqslant 0, \\
0 & \text { for } & 0<x_{n+1}<1, \\
x_{n+1}-1 & \text { for } & x_{n+1} \geqslant 1 .
\end{array}\right.
$$

Let $g_{n}^{*}$ be an arbitrary extension of $g_{n}$ to the homeomorphism of the plane onto itself. Consider the following inverse system of pairs of spaces:

$$
\left(E_{1}, X_{1}\right) \leftarrow \ldots \leftarrow\left(E_{n}, X_{n}\right) \leftarrow\left(E_{n}, Y_{n}\right) \leftarrow\left(E_{n+1}, X_{n+1}\right) \leftarrow \ldots
$$

where the arrows have the meaning described above.

The limit of the first membres is the plane. In fact, according to the remark at the beginning, the limit in question is the same as that of the system $E_{1} \leftarrow \ldots \leftarrow E_{n} \leftarrow E_{n+1}$ $\leftarrow \ldots$ with mappings equal to $\pi_{n}^{* n+1}$. That the last limit is the plane is an easy consequence of the geometrical form of mappings $\pi_{n}^{* n+1}$, which are constant with respect to the second coordinate and monotone with respect to the first one. Because the second members in the inverse system of pairs have continuum $X$ as the limit, the imbeddability of $X$ into the plane is proved.

Proof. Let $f_{1}, f_{2}, \ldots, f_{k}$ be all mappings in $\left[I^{\prime \prime} \rightarrow I^{\prime}\right]$. According to the uniformization theorem of [9], there exist mappings $\alpha_{1}, \alpha_{2}, \ldots, \alpha_{k}$ $\epsilon\left[I^{*} \rightarrow I^{\prime \prime}\right]$, where $I^{*}$ is a division of $I$, such that $f_{1} \alpha_{1}=f_{2} \alpha_{2}=\ldots=f_{k} \alpha_{k}$. Using again the uniformization theorem, we find a division $I^{\prime \prime \prime}$ of $I$ and mappings $\beta_{1}, \beta_{2}, \ldots, \beta_{k} \in\left[I^{\prime \prime \prime} \rightarrow I^{*}\right]$ such that $\alpha_{1} \beta_{1}=\alpha_{2} \beta_{2}=\ldots=\alpha_{k} \beta_{k}$. Let $g=\alpha_{i} \beta_{i}, i=1,2, \ldots, k$. We have $g \in\left[I^{\prime \prime \prime} \rightarrow I^{\prime \prime}\right]$. Mapping $g$ is the required strong majorant for $\left[I^{\prime \prime} \rightarrow I^{\prime}\right]$. In fact, we have for any $f_{i}$ and $f_{j}$ in question equalities $f_{i} g=f_{j} \gamma_{i, j}$, where $\gamma_{i, j}=\alpha_{j} \beta_{i}$. In other words $f_{j} \prec f_{i} g$ for any $f_{i}, f_{j} \in\left[I^{\prime \prime} \rightarrow I^{\prime}\right]$.

Remark. Factors $\gamma_{i, j}$ which realize inequalities $f_{j} \prec f_{i} g$ belong to $\left[I^{\prime \prime \prime} \rightarrow I^{\prime \prime}\right]$ as indicated in the proof of Lemma 1 .

Now, let $\varepsilon>0$. We write $f \underset{\varepsilon}{=} g$ if $|f(x)-g(x)| \leqslant \varepsilon$ for every $x \in I$. We prove in an elementary way the following approximation lemma:

Lemma 2. Let $f: I \rightarrow I$ be continuous and onto and let $\varepsilon>0$. There exists an integer $N$ such that for any divisions $I^{\prime}$ and $I^{\prime \prime}$ of $I$ such that $\eta\left(I^{\prime \prime}\right) / \eta\left(I^{\prime}\right) \geqslant N$ and mesh $I^{\prime} \leqslant \varepsilon / 4$ there exists a $g \in\left[I^{\prime \prime} \rightarrow I^{\prime}\right]$ such that $f=g$.

Proof. Let $f_{*}: I \rightarrow I$ be a simplicial mapping onto such that $f_{*}=f$. It satisfies the Lipschitz condition with a coefficient $K$, i.e. for every $x^{\prime}, x^{\prime \prime} \in I$ we have $\left|f_{*}\left(x^{\prime}\right)-f_{*}\left(x^{\prime \prime}\right)\right| \leqslant K\left|x^{\prime}-x^{\prime \prime}\right|$.

Let $I^{\prime}$ and $I^{\prime \prime}$ be divisions of $I$ such that $\eta\left(I^{\prime \prime}\right) / \eta\left(I^{\prime}\right) \geqslant N=[K]+1$ and such that mesh $I^{\prime} \leqslant \varepsilon / 4$. The first assumption implies that the image by $f_{*}$ of any segment of $I^{\prime \prime}$ lies in two adjacent segments of $I^{\prime}$.

It remains to define $g \in\left[I^{\prime \prime} \rightarrow I^{\prime}\right]$ which is an $\varepsilon / 2$-approximation of $f_{*}$. Let $0=e_{0}<e_{1}<\ldots<e_{s}=1$ be all division points in $I^{\prime \prime}$. Let $0=c_{0}<c_{1}$ $<\ldots<c_{t}=1$ be all division points in $I^{\prime}$. We define first a simplicial mapping $g_{*}$ by formula $g_{*}\left(e_{i}\right)=c_{k}$, where $c_{k}$ is the least division point in $I^{\prime}$ not less than $f_{*}\left(e_{i}\right)$. Mapping $g_{*}$ is not necessarily onto, but it may be improved in the following way.

Let $u, v \in I^{\prime \prime}$ be two adjacent points such that $f_{*}(u)=0$ and $f_{*}(v)=1$. We shall consider only the case $u<v$. If $u$ is one of division points of $I^{\prime \prime}$, then $g_{*}$ assumes value 0 . The same is true for $v$ and value 1 .

Then let $e_{j}<u<e_{j+1}$. We have $g_{*}\left(e_{j}\right)=g_{*}\left(e_{j+1}\right)=c_{1}$. We improve $g_{*}$ by defining a new mapping $g_{* *}$, also simplicial, by formulas

$$
g_{* *}\left(e_{k}\right)=\left\{\begin{array}{lll}
c_{m-1} & \text { for } & k \leqslant j, \text { where } c_{m}=g_{*}\left(e_{k}\right), \\
g_{*}\left(e_{k}\right) & \text { for } & k>j .
\end{array}\right.
$$

Let $e_{i}<v<e_{i+1}$. We have $g_{* *}\left(e_{i}\right)=g_{* *}\left(e_{i+1}\right)=c_{t-1}$. We improve $g_{* *}$ by defining a new mapping $g$, which is simplicial, by formulas

$$
g\left(e_{k}\right)= \begin{cases}c_{m+1} & \text { for } \quad k \geqslant i+1, \text { where } c_{m}=g_{* *}\left(e_{k}\right), \\ g_{* *}\left(e_{k}\right) & \text { for } \quad k \leqslant i\end{cases}
$$


Mapping $g$ is the required approximation of $f_{*}$, because, according to the construction and the remark at the beginning, for every $x \in I$ points $f_{*}(x)$ and $g(x)$ lie in two adjacent segments of $I^{\prime}$ and either of them has diameter not greater than $\varepsilon / 4$.

§ 2. A-categories. Let $X_{1}, X_{2}, \ldots$ be a sequence of closed unit intervals. Consider for every $X_{n}$ a sequence of divisions $\left\{X_{n, r}\right\}, r \geqslant n-1$, in the sense of $\S 1$. Assume that every $X_{n, r+1}$ is a proper subdivision of $X_{n, r}$, i.e. every segment of $X_{n, r+1}$ is at most a half of that of $X_{n, r}$. We have

$$
\lim _{r \rightarrow \infty} \operatorname{mesh} X_{n, r}=0 \quad \text { for every } \quad n=1,2, \ldots
$$

Consider classes of mappings $S_{m, n}^{r}=\left[X_{m, r} \rightarrow X_{n, r}\right]$ for all $n \leqslant m \leqslant r$ Assume that $S_{m, n}^{p} \subset S_{m, n}^{r}$ for $p \leqslant r$. A mapping $\varphi$ is said to be admissible if $\varphi \in\left[X_{m, m} \rightarrow X_{n, m}\right]=S_{m, n}^{m}=S_{m, n}$. It is easy to verify that the sequence $\left\{X_{n}\right\}$ and all admissible mappings form a category in the sense of [4]. This category is said to be an $A$-category if

$$
\lim _{r \rightarrow \infty} \eta\left(X_{r, r}\right) / \eta\left(X_{n, r}\right)=\infty \quad \text { for } \quad n=1,2, \ldots
$$

Property (2) implies the following approximation property:

(AP) Let an $\varepsilon>0$, an integer $n$ and a mapping $f: I \rightarrow I$ which is continuous and onto be given. There exists an $r_{0}$ such that for any $r \geqslant r_{0}$ there exists a mapping $g \in\left[X_{r, r} \rightarrow X_{n, r}\right]$ such that $f_{\varepsilon}=g$.

Proof. Take an $r_{0}$ such that mesh $X_{n, s} \leqslant \varepsilon / 4$ for $r \geqslant r_{0}$ (this is possible in virtue of (1)) and such that $\eta\left(X_{r, r}\right) / \eta\left(X_{n, r}\right) \geqslant N$, where $N$ depends on $f$ so that Lemma 2 is satisfied (this is possible in virtue of $(2)$ ).

Let $S_{m}=\bigcup_{n=1}^{m-1} S_{m, n}$. A mapping $g: I \rightarrow I$ will be said to be a special strong majorant for $S_{m}$ if it is a strong majorant for every $S_{m, n}, n=1, \ldots, m-1$.

LEMara 3. There exist a division $I^{*}$ of $I$ and mapping $g \in\left[I^{*} \rightarrow X_{m, m}\right]$ being a special strong majorant for $\mathrm{S}_{m}$.

Proof. Let $f_{m, n}$ be strong majorants for $S_{m, n}$. According to Lemma 1 they belong to $\left[I^{(n)} \rightarrow X_{m, n}\right]$, where $I^{(n)}$ is a division of $I$.

Applying the Remark to Lemma $1, f_{m, n}$ majorizes $\mathcal{S}_{m, n}$. with factors belonging also to $\left[I^{(n)} \rightarrow X_{m, m}\right]$.

Using the uniformization theorem we find mappings $\delta_{n} \in\left[I^{*} \rightarrow I^{(n)}\right]$, where $n=1,2, \ldots, m-1$, and $I^{*}$ is a division of $I$ such that $f_{m, 1} \delta_{1}=\ldots$ $=f_{m, m-1} \delta_{m-1}$. Denote by $g$ the last expressions. We have $g \in\left[I^{*} \rightarrow X_{m, m}\right]$. Mapping $g$ is the required special strong majorant for $S_{m}$. In fact, let $f^{\prime}, f^{\prime \prime} \in S_{m, n}$ for some $n \leqslant m-1$. As $f_{m, n}$ is the strong majorant for $S_{m, n}$, there exists a $\beta \in\left[I^{(n)} \rightarrow X_{m, m}\right]$ such that $f^{\prime} f_{m, n}=f^{\prime \prime} \beta$. Hence we have also $f^{\prime} f_{m, n} \delta_{n}=f^{\prime \prime} \beta \delta_{n}$, i.e. $f^{\prime} g=f^{\prime \prime} \gamma_{n}$, where $\gamma_{n}=\beta \delta_{n} \in\left[I^{*} \rightarrow X_{m, m}\right]$. This means that $f^{\prime} g \Leftarrow f^{\prime \prime}$. The lemma is proved.

Remark. Factors $\gamma_{n}$ which realize inequalities $f^{\prime} g \succ f^{\prime \prime}$ belong to $\left[I^{*} \rightarrow X_{m, m}\right]$, as indicated in the proof of Lemma 3 .

We say that an inverse system $\left\{X_{n}, \pi_{n}^{m}\right\}$ is contained in an $A$ - category if $X_{n}$ coincide with objects (in the sense of [4], p. 143) of this $A$-category and $\pi_{n}^{m}$ are admissible in it.

THEOREM 1. Every $\mathrm{SC}$ is an inverse limit of an inverse system contained in an arbitrary $A$-category $\mathfrak{A}$.

Proof. Let $X=\lim \left\{X_{n}, \sigma_{n}^{m}\right\}$ be an SC. Let $\left\{\varepsilon_{n}\right\}$ be a sequence of positive numbers with $\lim _{n \rightarrow \infty} \varepsilon_{n}=0$. We define the following diagram:

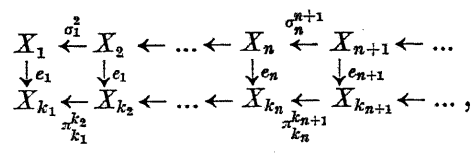

where $e_{1}, e_{2}, \ldots$ are identities and

$$
\begin{aligned}
& \pi_{k_{j}}^{k_{n}} e_{n} \sigma_{n}^{m} \underset{\varepsilon_{n}}{=} \pi_{k_{j}}^{k_{m}} e_{m}, \\
& \sigma_{j}^{n} e_{n}^{-1} \pi_{k_{n}}^{k_{m}} \underset{\varepsilon_{n}}{=} \sigma_{j}^{m} e_{m}^{-1},
\end{aligned}
$$

and the inverse system in the lower line is assumed to be in $\mathfrak{A}$.

We proceed by induction. Let $k_{1}=1$. Assume that $k_{j}$ for $j \leqslant n$ are already defined and that they have the properties required above. According to (AP), there exist $k_{n+1}$ and $\pi_{k_{n}}^{k_{n+1}} \epsilon\left[X_{k_{n+1}, k_{n+1}} \rightarrow X_{k_{n}, k_{n+1}}\right]$ such that the difference between $\sigma_{n}^{n+1}$ and $\pi_{k_{n}}^{k_{n+1}}$ is so small that the relations (4) and (5) hold for any $j \leqslant n$ and $m=n+1$.

The existence of diagram (3) is equivalent to the existence of a homeomorphism of $X$ onto the inverse limit of the inverse system in the lower line of (3) (see [1] and also [8]).

§ 3. Universal snake-like continua (USC). Let $\mathfrak{A}$ be an $A$-category. An SC is said to be universal in $\mathfrak{U}$ if it is an inverse limit of an inverse system $\left\{X_{n}, \pi_{n}^{m}\right\}$ contained in $\mathfrak{A}$ and such that every projection $\pi_{m}^{m+1}$ is a special strong majorant for $S_{m}$. We denote such an SC by USC (II).

We first prove some properties of USC (II) and then we establish its existence by construction.

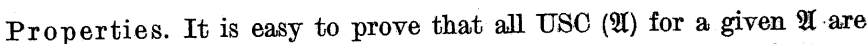
homeomorphic. However, the following more general theorem is true:

THEOREM 2. Alt USC are homeomorphic. 
Proof. Let $X=\lim \left\{X_{n}, \pi_{n}^{m}\right\}$ and $Y=\lim \left\{Y_{n}, \sigma_{n}^{m}\right\}$ be two USC, the first for the $A$-category $\mathfrak{U}^{\prime}$ and the second for the $A$-category $\mathfrak{U}^{\prime \prime}$ According to the theorem concerning homeomorphisms of inverse limits (see [1] and also [8]), in order to prove that $X$ and $Y$ are homeomorphic it is sufficient to show that for every $\varepsilon>0$, every $m$ and $n$, and every mapping $f_{m n}: X_{m} \rightarrow Y_{n}$ there exist $n^{\prime}>n$ and a mapping $g_{n^{\prime} m}: Y_{n^{\prime}} \rightarrow X_{m}$ such that

$$
f_{m n} g_{n^{\prime} m}=\sigma_{\varepsilon}^{n^{\prime}}
$$

and to show the same after substitution of $X$ for $Y$ and $Y$ for $X$.

To prove this, we choose an $\varepsilon$-approximation of $f_{m n}$ by a mapping $f_{m n}^{\prime} \in\left[Y_{r, r} \rightarrow Y_{n, r}\right]$, where $r>n$; such an approximation exists in virtue of Lemma 2. Let $n^{\prime}=r+1$. Because $\sigma_{r}^{r+1}$ is the strong majorant for $\left[Y_{r, r} \rightarrow Y_{n, r}\right]$, there exists a mapping $g_{n^{\prime} m}=g_{r+1, m} \in\left[Y_{r+1, r} \rightarrow Y_{r, r}\right]$ such that $f_{m n}^{\prime} g_{n^{\prime} m}=\sigma_{n}^{r} \sigma_{r}^{r+1}=\sigma_{n}^{n^{\prime}}$. Since $f_{m n}=f_{m n}^{\prime}$, the required $\varepsilon$-equality is proved.

The proof of a similar condition after the substitution of $X$ for $Y$ and $Y$ for $X$ is the same. Thus, the Theorem is proved.

Thanks to Theorem 2 we can henceforth write simply USC instead of $\operatorname{USC}(\mathfrak{X})$.

THEOREM 3. Every SC is a continuous image of USC.

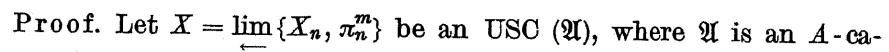
tegory. According to Theorem 1 , let $Y=\lim \left\{Y_{j_{n}}, \sigma_{j_{n}}^{j_{m}}\right\}$ be an arbitrary SC, where $\left\{Y_{j_{n}}, \sigma_{j_{n}}^{j_{m}}\right\}$ is an inverse system contained in $A$-category $\mathfrak{A}$. We define the commutative diagram

$$
\begin{aligned}
& X_{n_{1}} \leftarrow X_{n_{2}} \leftarrow \ldots \leftarrow X_{n_{k}} \leftarrow X_{n_{k+1}} \leftarrow \ldots \\
& \downarrow f_{1} \\
& \ddot{Y}_{j_{1}} \leftarrow{\stackrel{\downarrow}{j_{2}}}_{j_{2}} \leftarrow \ldots \leftarrow{\stackrel{\downarrow}{j_{k}}}_{j_{k}} \leftarrow{\stackrel{Y}{j_{k+1}}}_{j_{k+1}} \leftarrow \ldots
\end{aligned}
$$

where the vertical mappings are continuous and onto. It is known (see [4], p. 271 , Theorem 3.13 ) that such a diagram induces a continuous mapping
of $X$ onto $Y$.

We proceed by induction. Let $n_{1}=j_{1}+1$ and let $f_{1} \in S_{n_{1}}$. Assume that $n_{i}$ and $f_{i}$ are already defined for $i \leqslant k$, so that $n_{i}=j_{i}+1$ and $f_{i} \in S_{j_{i+1}}$. Let $n_{k+1}=j_{k+1}+1$. By the definition of USO ( $\mathfrak{U}$ ), the mapping $\pi_{n_{k+1}-1}^{n_{k+1}}$ $=\pi_{j_{k+1}}^{j_{k+1}+1}$ is a special strong majorant for $S_{j_{k+1}}$. Hence (see Lemma 3 and Remark after it) there exists a mapping, which we denote by $f_{k+1}$, belonging to the class $S_{n_{k+1}}=S_{j_{k+1}+1}$ and such that $\sigma_{j_{k}}^{j_{k+1}} f_{k+1}=f_{k} \pi_{n_{k}}^{n_{k+1}}$ (= $f_{k} \pi_{n_{k}}^{n_{k+1}-1} \pi_{n_{k+1}-1}^{n_{k+1}}$ ). Thus, the theorem is proved.

Construction. We shall define an $A$-category $\mathfrak{A}$ and an $\operatorname{USC}(\mathfrak{U})$ simultaneously.
Let $X_{0}$ and $X_{1}$ be two unit closed intervals with divisions $X_{0,1}$ and $X_{1,1}$ consisting of 2 and 4 segments respectively. Let $\pi_{1}^{2}$ be a strong majorant for $S_{1}=\left[X_{3,1} \rightarrow X_{0,1}\right]$. It is in class $\left[X_{2,1} \rightarrow X_{1,1}\right]$, where $X_{2,1}$ is a division of the unit closed interval $X_{2}$. Consider a subdivision $X_{2,2}$ of $X_{2,1}$ created from $X_{2,1}$ by dividing all segments in $X_{2,1}$ into three equal parts. The subdivision $X_{2,2}$ induces subdivisions $X_{2,1}, X_{0,2}$ of $X_{1,1}$ and $X_{0,1}$ created by simplicial images from $X_{2,1}$ onto $X_{1,1}$ and then onto $X_{0,1}$. We have immediate inclusions $S_{m, n}^{p} \subset S_{m, n}^{r}$, if $p<r$ for the integers in question. We have also $\pi_{1}^{2} \in S_{2,1} \subset S_{2}$.

Assume that divisions $X_{p, r}$ of $X_{p}$ and mappings $\pi_{p-1}^{n}$ are already defined for $p, r \leqslant n$ and $r \geqslant p-1$, and that they have the following properties:

(i) $\pi_{m}^{p} \in\left[X_{p, p} \rightarrow X_{m, p}\right]=S_{p, m}$ for $m \leqslant p$,

(ii) $\pi_{p-1}^{p}$ is a special strong majorant for $S_{p-1}, p \leqslant n$,

(iii) $S_{p, m}^{r} \subset S_{p ; m}^{q}$ for $r<q \leqslant n$ and $m<p \leqslant n$.

We choose now $\pi_{n}^{n+1}$ as a special strong majorant for $S_{n}$. According to Lemma 3 , we have $\pi_{n}^{n+1} \in\left[X_{n+1, n} \rightarrow X_{n, n}\right]$, where $X_{n+1, n}$ is a division of $X_{n+1}$. Consider a subdivision $X_{n+1, n+1}$ of $X_{n+1, n}$ created from $X_{n+1, n}$ by dividing all segments of $X_{n+1, n}$ into three equal parts. The subdivision $X_{n+1, n+1}$ induces subdivisions $X_{p, n+1}$ of $X_{p, n}, p=0,1, \ldots, n$, in the same way as $X_{2,2}$ induces $X_{1,2}$ and $X_{0,2}$. We then have $\pi_{p}^{n+1} \subset S_{n+1, p}$ for $p \leqslant n$, and inclusions $S_{p, m}^{r} \subset S_{p, m}^{r+1}$ for $r \leqslant n+1$ and $m \leqslant p \leqslant n+1$. Thus, the construction is finished.

We shall prove in $\S 5$ that the category constructed is an $A$-category.

§ 4. An estimation. We still consider the category constructed in $\S 3$.

LEMMA 4. $\eta\left(X_{n, n}\right)-\eta\left(X_{n-1, n}\right) \geqslant 2$ implies $\eta\left(X_{n+1, n}\right) \geqslant 3 \eta\left(X_{n, n}\right)$.

Proof. Let $\sigma_{n}$ be a segment of $X_{n, n}$. Let $f^{\prime}, f^{\prime \prime} \in\left[X_{n, n} \rightarrow X_{n-1, n}\right]$ be two mappings defined as follows. We define $f^{\prime}$ as a mapping which maps $\sigma_{n}$ onto a segment $\sigma_{n-1}$ of $X_{n-1, n}$ and

$$
f^{\prime-1}\left[\operatorname{Int}\left(\sigma_{n-1}\right)\right]=\operatorname{Int}\left(\sigma_{n}\right)
$$

Such a mapping exists, as $\eta\left(X_{n, n}\right) \geqslant \eta\left(X_{n-1, n}\right)$.

From $\eta\left(X_{n, n}\right)-\eta\left(X_{n-1, n}\right) \geqslant 2$ it follows that there exist three adjacent segments $\sigma_{n}^{1}, \sigma_{n}^{2}$ and $\sigma_{n}^{3}$ of $X_{n, n}$ and a mapping $f^{\prime \prime} \in\left[X_{n, n} \rightarrow X_{n-1, n}\right]$ such that

$$
f^{\prime \prime}\left(\sigma_{n}^{i}\right)=\sigma_{n-1}, \quad i=1,2 \text { and } 3
$$


Since $\pi_{n}^{n+1}$ is a special. strong majorant for $S_{n}$, there exists an $a \in\left[X_{n+1, n} \rightarrow X_{n, n}\right]$ such that the diagram

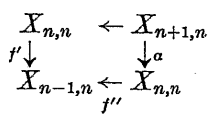

is commutative.

Let $\sigma_{n+1}^{1}, \sigma_{n+1}^{2}$ and $\sigma_{n+1}^{3}$ be segments of $X_{n+1, n}$ such that $\alpha\left(\sigma_{n+1}^{i}\right)=\sigma_{n}^{i}$ for $i=1,2$ and 3. By (10) and the commutativity of (11), we have $f^{\prime} \pi_{n}^{n+1}\left(\sigma_{n+1}^{i}\right)=\sigma_{n-1}$ for $i=1,2$ and 3 . From this equality and (9) we obtain $\pi_{n}^{n+1}\left(\sigma_{n+1}^{i}\right)=\sigma_{n}, i=1,2$ and 3 . Hence every segment $\sigma_{n}$ of $X_{n, n}$ is an image by $\pi_{n}^{n+1}$ of at least three segments of $X_{n+1, n}$. Thus, $\eta\left(X_{n+1, n}\right)$ $\geqslant 3 \eta\left(X_{n, n}\right)$.

\$ 5. The proof of property (2). The first members of the implication in Lemma 4 are true for all $n$. In fact, this is valid for $n=1$, as $\eta\left(X_{1,1}\right)=4$ and $\eta\left(X_{0,1}\right)=2$. Suppose that this is valid for every $n \leqslant k$. According to Lemma 4, we have $\eta\left(X_{k+1, k}\right) \geqslant 3 \eta\left(X_{k, k}\right)$ and, by the definition of subdivisions described in the construction (see $\S 3$ ), we have $\eta\left(X_{k+1, k+1}\right) \geqslant 3 \eta\left(X_{k, k+1}\right)$. Therefore, $\eta\left(X_{k+1, k+1}\right)-\eta\left(X_{k, k+1}\right) \geqslant 2 \eta\left(X_{k, k+1}\right)$. It is obvious that $\eta\left(X_{k, k+1}\right) \geqslant 12$ for $k \geqslant 1$. Thus, $\eta\left(X_{k+1, k+1}\right)-\eta\left(X_{k, k+1}\right)$ $\geqslant 24>2$.

Hence, also the second members of the implication are true for all $n$. We obtain for $n+j \leqslant r$

$$
\eta\left(X_{n+j, r}\right) / \eta\left(X_{n+j-1, r}\right)=\eta\left(X_{n+j, n+j}\right) / \eta\left(X_{n+j-1, n+j}\right) \geqslant 3 .
$$

Finally, we have

$\eta\left(X_{r, r}\right) / \eta\left(X_{n, r}\right)=\eta\left(X_{n+1, r}\right) \eta\left(X_{n+2, r}\right) \ldots \eta\left(X_{r, r}\right) / \eta\left(X_{n, r}\right) \times$

$$
\times \eta\left(X_{n+1, r}\right) \ldots \eta\left(X_{r-1, r}\right) \geqslant 3^{r-n-1} .
$$

This ends the proof of (2).

Because all the properties of $A$-categories, except (2), are assumed in the construction, the proof of the existence of USC is finished.

§ 6. Other properties of USC. Let $I^{\prime}$ and $I^{\prime \prime}$ be divisions of the closed unit interval $I$. Let $f \in\left[I^{\prime \prime} \rightarrow I^{\prime}\right]$. Let $J$ be a subinterval of $I^{\prime}$. We say that $f$ has a full oscillation over $J$ if, for every $J^{\prime} \subset J, J^{\prime} \neq J$, where $J^{\prime}$ is also a subinterval of $I^{\prime}$, and for every component $C$ of $f^{-1}(J)$ such that $C$ is an interior subinterval of $I^{\prime \prime}$ and $f$ maps the ends of $C$ onto the ends of $J$, there exist at least two intervals $C_{1}, C_{2} \subset C$ such that $f\left(C_{1}\right)=f\left(C_{2}\right)=J^{\prime}$. This notion of oscillation is parallel to that of crookedness in [2].

THeOREM 4. If $X=\lim _{\leftarrow}\left\{X_{n}, \pi_{n}^{m}\right\}$ is an USC, then every $\pi_{n}^{n+1}, n>1$, has full oscillation over any subinterval $J$ of $X_{n, n}$.
Proof. Let $J^{\prime} \subset J, J^{\prime} \neq J$, be a subinterval of $X_{n, n}$. We shall define the commutative diagram

$$
\begin{gathered}
\left(X_{n, n}, J\right) \leftarrow\left(X_{n+1, n}, K\right) \\
\quad f^{\prime} \downarrow \\
\left(X_{n-1, n}, L\right) \leftarrow\left(\begin{array}{c}
\downarrow^{\alpha} \\
f^{\prime \prime}
\end{array}\left(X_{n, n}, M\right)\right.
\end{gathered}
$$

We define $f^{\prime} \in\left[X_{n, n} \rightarrow X_{n-1, n}\right]$ as a monotone mapping which maps $J$ and $J^{\prime}$ onto subintervals $L$ and $L^{\prime}$ of $X_{n-1, n}$ respectively and such that

$$
f^{\prime-1}(L)=J \quad \text { and } \quad f^{\prime-1}\left(L^{\prime}\right)=J^{\prime} .
$$

Such a mapping always exists if $\eta\left(X_{n-1, n}\right) \geqslant 5$, which is true for $n>1$; the existence is a consequence of inequality $\eta\left(X_{n, n}\right) \geqslant \eta\left(X_{n-1, n}\right)$.

We define $f^{\prime \prime} \epsilon\left[X_{n, n} \rightarrow X_{n-1, n}\right]$ as a mapping which maps an interval $M$ of $X_{n, n}$ onto $L$ with full oscillation over $L$ and such that

$$
f^{\prime \prime-1}(L)=M \text {. }
$$

Such a mapping exists according to the estimation $\eta\left(X_{n, n}\right) \geqslant$ $\geqslant 3 \eta\left(X_{n-1, n}\right)$, which follows from Lemma 4 .

Since $\pi_{n}^{n+1}$ is a special strong majorant for $S_{n}$, there exists an $a \epsilon\left[X_{n+1, n} \rightarrow X_{n, n}\right]$ filling up the diagram.

Consider a component $K$ of $\left(\pi_{n}^{n+1}\right)^{-1}(J)$ such that $K$ is an interior subinterval of $X_{n+1, n}$ and such that $\pi_{n}^{n+1}$ maps the ends of $K$ onto the ends of $J$. We have $\pi_{n}^{n+1}(K)=J$. We prove that

$$
\alpha(K)=M \text {. }
$$

In fact, if $x \in K$, then $\alpha(x) \in M$, because in the other case $f^{\prime \prime} \alpha(x) \epsilon L$ in virtue of $(15)$, Since $f^{\prime} \pi_{n}^{n+1}(x) \in L$, we have a contradiction of the commutativity of diagram (13).

Note that $\alpha$ maps the ends $a^{\prime}$ and $a^{\prime \prime}$ of $K$ into the ends of $M$. In fact, if $\alpha\left(a^{\prime}\right) \in \operatorname{Int}(M)$, then, because $K$ is interior in $X_{n+1}$, there exists an $x \in X_{n+1}-K$ such that $\alpha(x) \in M$ and $\pi_{n}^{n+1}(x) \xi J$. This implies $f^{\prime \prime} \alpha(x) \in L$ and, by (14), $f^{\prime} \pi_{n}^{n+1}(x) \notin L$. We have a contradiction of the commutativity of diagram (13).

We have, in addition, $\alpha\left(a^{\prime}\right) \neq \alpha\left(a^{\prime \prime}\right)$, because in the other case $f^{\prime} \pi_{n}^{n+1}\left(a^{\prime}\right)=f^{\prime} \pi_{n}^{n+1}\left(a^{\prime \prime}\right)$, by (13). This contradicts the definition of $K$ and $f^{\prime}$. Thus, the equality $a(K)=M$ is proved.

By the definition of $f^{\prime \prime}$, the inverse image $f^{\prime \prime-1}\left(L^{\prime}\right) \cap M$ consists of at least two components which are mapped by $f^{\prime \prime}$ onto $L^{\prime}$. Hence, $\left(f^{\prime \prime} \alpha\right)^{-1}\left(L^{\prime}\right) \cap K$ and, by the commutativity of (13), also $\left(f^{\prime} \pi_{n}^{n+1}\right)^{-1}\left(L^{\prime}\right) \cap K$ contains at least two components which are mapped by $f^{\prime \prime} \alpha$ and $f^{\prime} \pi_{n}^{n+1}$, respectively, onto $L^{\prime}$. We have, by $(14),\left(f^{\prime} \pi_{n}^{n+1}\right)^{-1}\left(L^{\prime}\right)=\left(\pi_{n}^{n+1}\right)^{-1} f^{\prime-1}\left(L^{\prime}\right)$ 
$=\left(\pi_{n}^{n+1}\right)^{-1}\left(J^{\prime}\right)$. Hence, $\left(\pi_{n}^{n+1}\right)^{-1}\left(J^{\prime}\right)$ contains in $K$ at least two components which are mapped by $\pi_{n}^{n+1}$ onto $J^{\prime}$. Thus, $\pi_{n}^{n+1}$ has full oscillation over $J$.

Let $A=\lim _{\leftarrow}\left\{A_{n}, \pi_{n}^{\prime m}\right\}$, where $A_{n} \subset X_{n}$ are closed intervals and $\pi_{n}^{\prime m}$ $=\pi_{n}^{m} \mid A_{m}$, be a subcontinuum of $X$.

LEMMA 5. Let $A_{n}^{\prime}$ be the maximal interval of $X_{n, n}$ contained in the interior of $A_{n}$. If $\pi_{n}^{\prime n+1}$ has, for every $n$, full oscillation over $A_{n}^{\prime}$, then $A$ is indecomposable.

Proof. Let $B=\varliminf_{\longleftarrow}\left\{B_{n}, x_{n}^{m}\right\}$, where $B_{n} \subset A_{n}$ are closed intervals and $\varkappa_{n}^{m}=\pi_{n}^{\prime m} \mid B_{m}$, be a proper subcontinuum of $A$. We prove that $B$ is non-dense in $A$. We can assume, without loss of generality, that $A_{n}-B_{n}$ $\neq 0$ and that any non-empty component of $A_{n}-B_{n}$ contains at least two segments of $X_{n, n}$. We then have $B_{n} \subset A_{n}^{\prime}$ and $B_{n} \neq A_{n}^{\prime}$.

As $A_{n}^{\prime}$ lies in the interior of $A_{n}$ and $\pi_{n}^{\prime n+1}$ is onto, there exists a conponent $C$ of $\left(\pi_{n}^{\prime n+1}\right)^{-1}\left(A_{n}^{\prime}\right)$ such that $C$ lies in the interior of $A_{n+1}$ and $\pi_{n}^{\prime n+1}$ maps the ends of $C$ onto the ends of $A_{n}^{\prime}$. Let $A_{n}^{\prime \prime}$ be an interval of $X_{n, n}$ which is a proper subinterval of $A_{n}^{\prime}$. Note that if $b_{n} \in B_{n}$, then

$$
\varrho\left(b_{n}, A_{n}^{\prime \prime}\right) \leqslant \operatorname{mesh} X_{n, n} .
$$

As $\pi_{n}^{\prime n \div 1}$ has full oscillation over $A_{n}^{\prime}$, there exist disjoint intervals $C_{1}$ and $C_{2}$ in $C$ such that $\pi_{n}^{\prime n+1}$ maps $C_{1}$ and $C_{2}$ onto $A_{n}^{\prime \prime}$. As $B_{n} \subset A_{n}^{\prime}$ and $B_{n} \neq A_{n}^{\prime}, B_{n+1}$ is disjoint with one of these intervals. Denote this interval by $C^{n+1}$. Let $D^{n+1}=\pi_{n+1}^{\prime-1}\left(C^{n+1}\right) \subset A$ where $\pi_{n+1}^{\prime}=\pi_{n+1} \mid A$. We have $D^{n+1}$ $\cap B=0$ for every $n$ in question.

Let $b \in B$. For every $n$ there exists a point $d^{n+1} \in D^{n+1}$ such that

(17) $\varrho\left(b, d^{n+1}\right) \leqslant \sum_{k=1}^{n}\left(1 / 2^{k}\right) \cdot \operatorname{mesh} X_{k, n}+\sum_{k=n+1}^{\infty} 1 / 2^{k} \leqslant n / 3^{n-1}+1 / 2^{n}$.

In order to find $d^{n+1}$, we choose $b_{n}^{\prime} \in A_{n}$ such that $\left|b_{n}^{\prime}-b_{n}\right| \leqslant \operatorname{mesh} X_{n, n}$ and such that the set

$$
\left(\pi_{n}^{\prime n+1}\right)^{-1}\left(b_{n}^{\prime}\right) \frown C^{n+1}
$$

is non-empty. Such a $b_{n}^{\prime}$ exists according to (16). We define $d^{n+1}$ as a point in $\pi_{n+1}^{-1}$ of the set (18). We have $\lim _{n \rightarrow \infty} d^{n}=b$, in virtue of (17). Thus, $B$ is non-dense in $A$, since $d^{n} \notin B$.

THEOREM 5. USC is a hereditarily indecomposable continuum $\left({ }^{2}\right)$.

Proof. Let $X$ be an USC and let $A=\lim _{\longleftarrow}\left\{A_{n}, \pi_{n}^{\prime m}\right\}$ be a subcontinuum of $X$. We shall prove that $A$ is indecomposable.

(2) So is the pseudoarc; see [2], [6], and [10].
Let $A_{n}^{\prime}$ be the maximal interval of $X_{n, n}$ contained in the interior of $A_{n}$. There exists a component $C$ of $\left(\pi_{n}^{n+1}\right)^{-1}\left(A_{n}^{\prime}\right)$ such that $C$ is in the interior of $A_{n+1}$ and such that $\pi_{n}^{n+1}$ maps the ends of $C$ onto the ends of $A_{n}^{\prime}$. Let $A_{n}^{\prime \prime}$ be a proper subinterval (in $X_{n, n}$ ) of $A_{n}^{\prime}$. According to Theorem 4 , $\pi_{n}^{n+1}$ has full oscillation over $A_{n}^{\prime}$. Hence there exist intervals $C_{1}$ and $C_{2}$ in $C$ disjoint and such that $\pi_{n}^{n+1}$ maps those intervals onto $A_{n}^{\prime \prime}$. Because $C \subset A_{n+1}$, intervals $C_{1}$ and $C_{2}$ also lie in $A_{n+1}$. Hence, the partial mapping $\pi_{n}^{\prime n+1}=\pi_{n}^{n+1} \mid A_{n+1}$ also has full oscillation over $A_{n}^{\prime}$. Then, in virtue of Lemma 5 , the subcontinuum $A$ is indecomposable. Thus, the theorem is proved.

\section{References}

[1] P. Alexandroff, Untersuchungen über Gestalt und Lage abgeschlossener Mengen beliebieger Dimension, Ann. of Math. 30 (1929), pp. 101-187.

[2] R. H. Bing, A homogeneous indecomposable plane continuum, Duke Math. Journ. 15 (1948), pp. 729-742.

[3] - Concerning hereditarily indecomposable continua, Pacific Jour. Math. 1 (1951), pp. 43-51.

[4] S. Eilenberg and N. Steenrod, Foundations of algebraic topology, Princeton 1952 .

[5] I. R. Isbell, Embedding of inverse limits, Ann. of Math, 70 (1959), pp. 73-84. [6] B. Knaster, Un continu dont tout sous-continu est indécomposable, Fund.
Math. 3 (1922), pp. 247-286. 3 (1922), pp. 247-286.
[7] G. R. Lehner, Extent

Math. Soc. 98 (1961), pp. 369-394.

[8] J. Mioduszewski, Mappings of inverse limits, Coll. Math. 9, in preparation.

[9] - On a quasi-ordering in the class of continuous mappings of the closed interval onto itself, Coll. Math. 9 (1962), in preparation.

[10] E. E. Moise, An indecomposable plane continuum which is homeomorphic to each of its nondegenerate subcontinua, Trans. Amer. Math. Soc. 63 (1954), pp. 581-594.

[11] R. Sikorski and K. Zarankiewicz, On uniformization of functions (I), Fund. Math. 41 (1954), pp. 339-344.

[12] Z. Waraszkiewicz, Sur quelques invariants des transformations continues, Fund. Math. 23 (1934), pp. 172-189.

INSTYTUT MATEMATYCZNY UNIWERSYTETU WROCEAWSKIEGO
THE MATHEMATICAL INSTITUTE OF WROCEAW UNIVERSTTY

Reçu par la Rédaction le 25. 9. 1961 